\section{Using the SMART ${ }^{\mathrm{TM}}$ cDNA System to Map the Transcription Initiation Site}

BioTechniques 28:_-_(May 2000)

Primer extension is a common method used to determine the transcription initiation site of a gene by determining the $5^{\prime}$ end of the mRNA. A frequently encountered problem of this assay is that the reverse transcriptase pauses or terminates at various sites before reaching the $5^{\prime}$ end of the mRNA, resulting in multiple extension products. Under these circumstances, the real transcription start site cannot be easily determined. This premature termination of the reverse transcriptase could be from various reasons such as the secondary structure of the mRNA. Because of this, primer extension assays require a primer quite close to the $5^{\prime}$ end of the mRNA (preferably within 100 bases). In addition, when the gene of interest is expressed at low levels, a large amount of total RNA or even mRNA is needed because of the limited sensitivity of this assay. These difficulties often make a simple primer extension assay into one of the most tedious and difficult assays to perform in the laboratory.

During our attempt to map the transcription initiation site of the zebrafish vascular endothelial growth factor gene (vegf), we encountered many of these difficulties. The zebrafish vegf mRNA is present at a very low level in adult total RNA, and there is a very long and extremely AT rich $5^{\prime}$ untranslated region. We designed a few primers from the estimated $5^{\prime}$ end of the mRNA (from Northern analysis data) and used them in primer extension assays. No extension products were obtained when avian myeloblastosis virus (AMV) reverse transcriptase (Promega, Madison, WI, USA) was used (data not shown). When SUPERSCRIPT II ${ }^{\mathrm{TM}}$ reverse transcriptase (Life Technologies, Rockville, MD, USA) was used, multiple extension products appeared (data not shown). To solve this problem, we tried a PCR method based on the templateswitching effect of the reverse transcriptase (the SMART ${ }^{\mathrm{TM}}$ cDNA synthesiscoupled PCR method) to determine the $5^{\prime}$ end of the mRNA (Clontech Laboratories, Palo Alto, CA, USA) (Figure 1) (1-3). The result was then confirmed by a primer extension assay using a primer located at about 70 bases downstream of the determined $5^{\prime}$ end of the mRNA.

A gene-specific primer, GS1 (4 pmol; 5'-AAACTAAAAACACACACAAA$\left.3^{\prime}\right)$, was used to initiate the first-strand cDNA synthesis for $1 \mathrm{~h}$ at $42^{\circ} \mathrm{C}$ using 4 $\mu \mathrm{g}$ adult total RNA or 24-h embryonic total RNA as the template and SUPERSCRIPT II reverse transcriptase. When the reverse transcriptase reaches the $5^{\prime}$ end of the mRNA, its terminal transferase activity added a few additional $\mathrm{dCs}$ to the $3^{\prime}$ end of the first-strand cDNA $(2,3)$. The SMART II oligonucleotide (5'-AAGCAGTGGTATCAACGCAGAGTACGCGGG-3'), which is included in the same reaction then paired with the tail Cs and caused a template switching of the reverse transcriptase. The reverse transcriptase then extends to the end of the SMART II oligonucleotide (Figure 1). Next, RNase H (Life Technologies) was used to digest the RNA in the DNA-RNA hybrid. Only the full-length, first-strand cDNA molecules containing the ex-

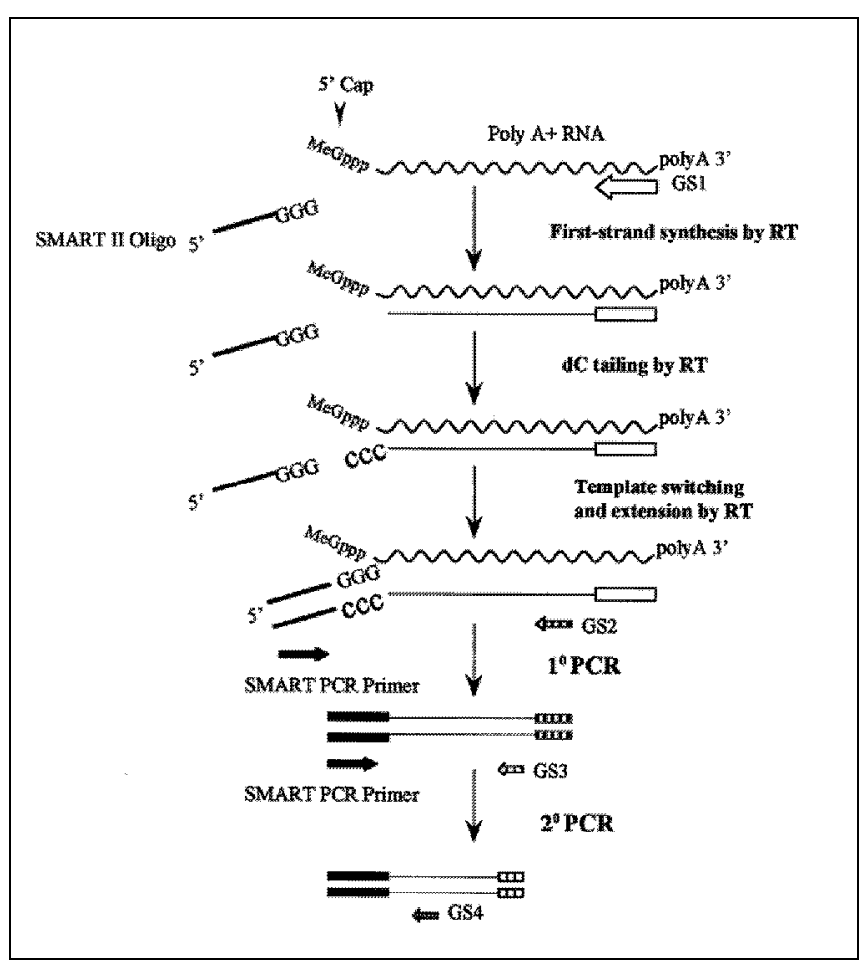

Figure 1. The outline of the strategy of the SMART cDNA PCR amplification method used in this work. GS1-4 represent gene-specific primers 1-4.

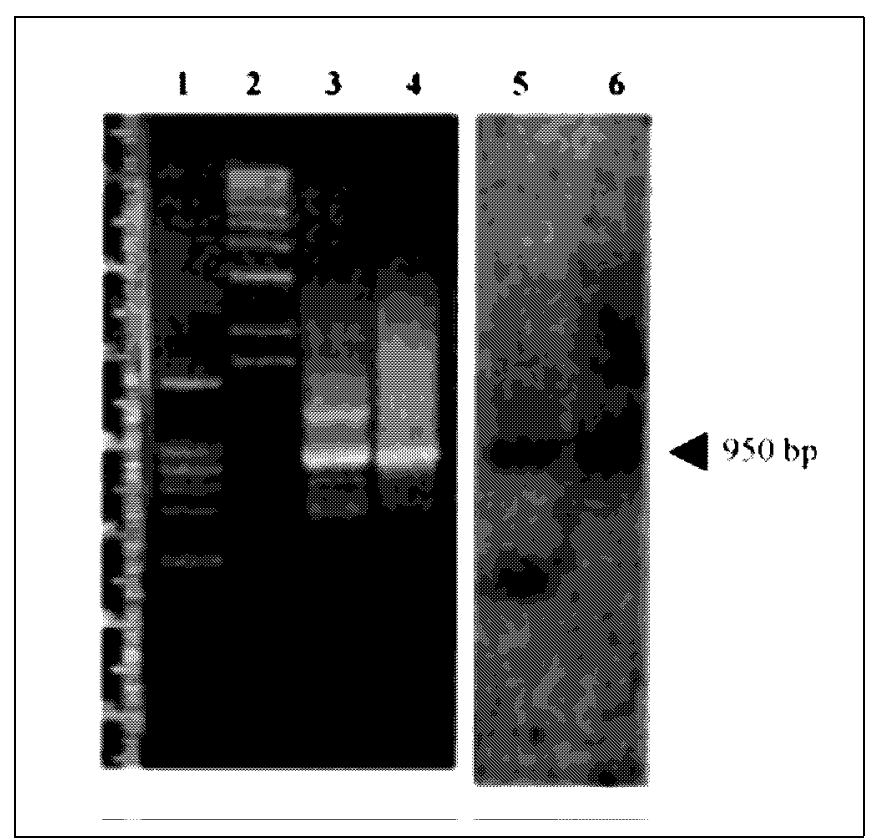

Figure 2. Agarose gel of the vegf-specific PCR products from the secondary reactions described in Figure 1. Lanes 1 and 2, molecular weight markers; lanes 3 and 4, secondary PCR products from GS3 and SMART PCR primers from $4 \mu \mathrm{g}$ total RNA isolated from adult zebrafish or 24-h post-fertilization embryos; lanes 5 and 6, Southern analysis of DNA from lanes 3 and 4 using labeled GS4 as probe. 
tended Cs can serve as the template for the subsequent PCR amplification. A small aliquot of the above cDNA was then amplified by primary PCR using the SMART PCR primer (5'-AAGCAGTGGTATCAACGCAGAGT-3') and a second gene-specific primer, GS2 (5'GTTCGCTCGATCATCTTGGC-3'). No discrete bands were observed from the primary reactions (data not shown). Finally, secondary PCR was carried out using the same SMART PCR primer together with a third gene-specific primer GS3 (5'-CGGGACGCATTCGAGCGCCTCATC-3').

While the adult total RNA gave a few PCR product bands, including the dominant 950-bp band, the 24-h embryonic total RNA only gave one prominent band of $950 \mathrm{bp}$. To confirm that this 950-bp band is a vegf-specific PCR product, the gel was blotted and Southern hybridization was performed using the fourth gene-specific primer, GS4 (5'-CTGGTTCGTACTGCCGTGG-3') as probe (Figure 2). As shown in Figure
2, the 950-bp PCR product is the longest vegf-specific product, indicating that the transcription initiation site of this gene is most likely residing at about $950 \mathrm{bp}$ upstream from primer GS3. To determine the transcription initiation site more precisely, we directly sequenced the 950-bp PCR products using a gene-specific primer (5'-CTATAGGGCCAGAGGACGG-3') (Figure $3 \mathrm{~A})$. This sequencing ends at the end of the SMART II oligonucleotide (Figure 3A). Starting a few nucleotides from the SMART II oligonucleotide, the sequencing showed an area of mixed peaks, possibly from the variation of the number of Cs added at the $5^{\prime}$ ends of the first-strand cDNA. Nevertheless, the SMART II oligonucleotide sequence can still be identified unambiguously.

We also cloned the PCR products into pGEM $^{\circledR}$-T vector (Promega, Madison, WI, USA) and sequenced two clones using primers from the polylinker of the vector. The two clones gave the same sequence as shown in Figure
3B. By comparing the sequences from the PCR cDNA with sequences of the $5^{\prime}$ flanking genomic DNA, we can identify the $5^{\prime}$ nucleotide of the mRNA to be either the $\mathrm{C}$ ( $\mathrm{G}$ in mRNA) before the stretch of 3Cs from the SMART II oligonucleotide or the next nucleotide $\mathrm{T}$ (A in the mRNA) before the stretch of 4Cs (Figure 3B).

To confirm the accuracy of the above transcription initiation site, we designed a few new primers that are within 100 bases of the $5^{\prime}$ end of the vegf mRNA for the primer extension assay. The primer GS4, which is located at about 70 basesfrom the transcription initiation site, worked well in primer extensions. The primer extension reactions were conducted using either the heat-stable ThermoScript ${ }^{\mathrm{TM}}$ reverse transcriptase at $65^{\circ} \mathrm{C}$ or using SUPERSCRIPT II reverse transcriptase at $42^{\circ} \mathrm{C}$ (Life Technologies, Rockville, MD, USA) (4). As shown in Figure 4A, the high-temperature primer extension using ThermoScript reverse transcriptase resulted in a

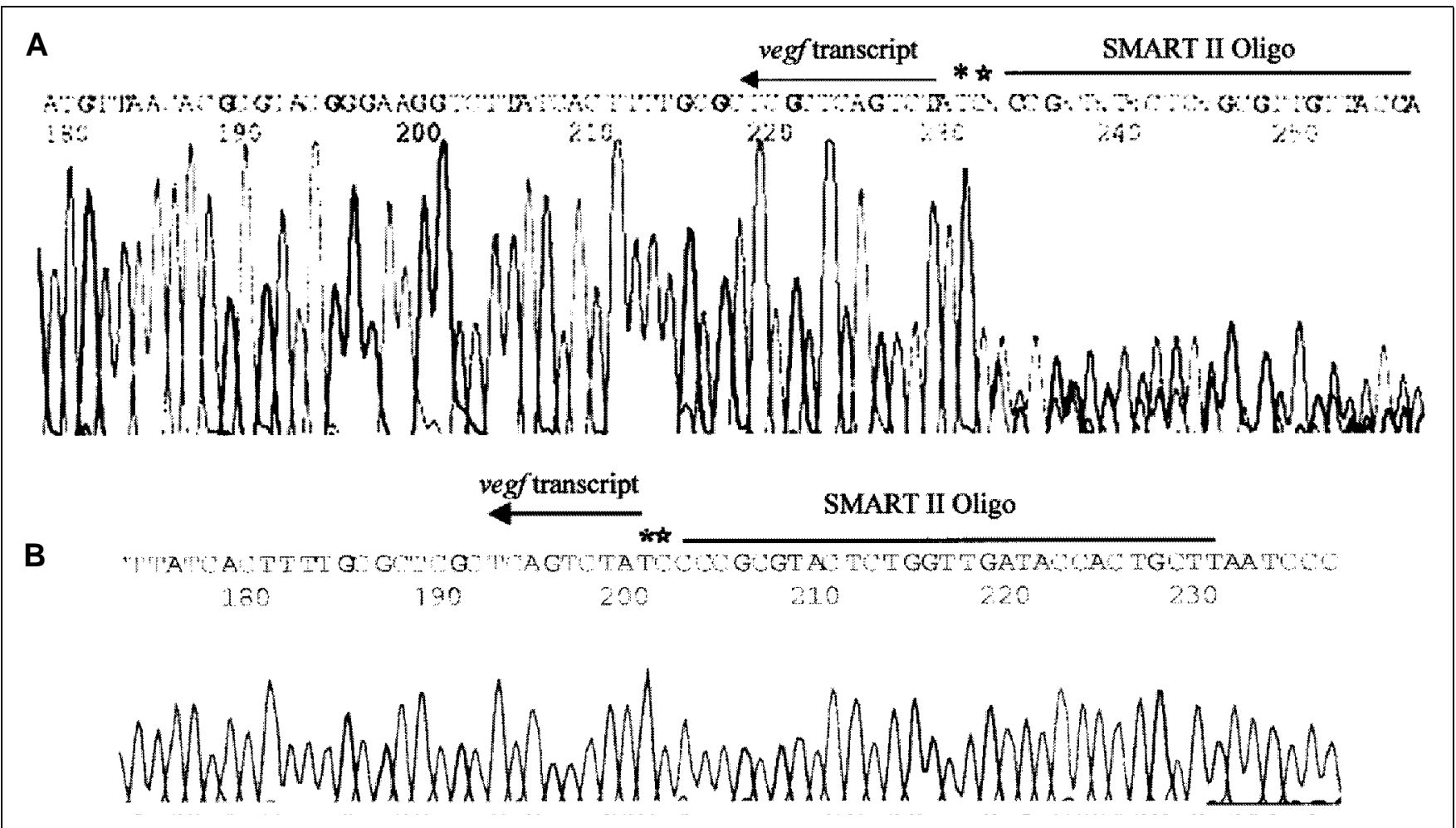

Figure 3. Determination of the transcription initiation site using the SMART cDNA PCR method. (A) Direct sequencing of the SMART PCR 950-bp product. The possible transcription initiation sites are indicated by stars. The vegf mRNA sequence starts from the arrow, and the SMART II oligonucleotide sequence is indicated by the line above the sequence. (B) Sequencing of a pGEM-T plasmid containing the SMART PCR 950-bp product. The possible transcription initiation sites are indicated by stars. The vegf mRNA sequence starts from the arrow, and the SMART II oligonucleotide sequence is indicated by the line above the sequence. 
single clean extended product, while SUPERSCRIPT II reverse transcriptase generated two extended products. The dominant band from SUPERSCRIPT II reverse transcriptase is identical to the ThermoScript reverse transcriptase band (Figure 4A, lanes 1 and 2). When the ThermoScript primer extension products were run alongside a sequencing reaction using the same primer and genomic DNA clones, the transcription initiation site was mapped to AT nucleotides (UA in the mRNA). This $\mathrm{T}$ is identical to the $\mathrm{T}$ determined by the

SMART PCR method above (compare Figure 3B and Figure 4B).

It is important to mention that several primers located within 100 bases of the $5^{\prime}$ end of the mRNA did not work well in primer extension assays in our hands. We had to screen multiple primers to find a suitable primer for the primer-extension assay of our gene. This is another example of the possible complexity of performing primer extension assays.

The second discrete band generated by SUPERSCRIPT II reverse transcriptase is about 15 bases longer than the dominant band (Figure 4A, lane 2). This additional extension product by SUPERSCRIPT II reverse transcriptase may reflect a second transcription initiation site of the vegf gene in zebrafish. However, both the SMART cDNA synthesis PCR (SUPERSCRIPT II reverse transcriptase used in first-strand cDNA synthesis) as well as primer-extension using ThermoScript reverse transcriptase did not detect this site. In addition, we noticed that SUPERSCRIPT II reverse transcriptase yielded many more primerextended products compared with ThermoScript reverse transcriptase (Figure 4A). This seems to reflect the different level of activity of the two reverse transcriptases. While ThermoScript is derived from AMV reverse transcriptase, SUPERSCRIPT II is derived from Moloney murine leukemia virus (MMLV) reverse transcriptase.

Figure 4. Determination of the transcription initiation site by primer extension. (A) Primer extension using ThermoScript reverse transcriptase (lane 1) and SUPERSCRIPT II reverse transcriptase (lane 2) using primer GS4. The bold arrow on the right indicates the dominant primer extension product of SUPERSCRIPT II reverse transcriptase and ThermoScript reverse transcriptase. The thin arrow indicates the second primer extension product by SUPERSCRIPT II reverse transcriptase. (B) The primer-extended product from ThermoScript reverse transcriptase was run alongside the genomic DNA clones sequenced using a gene-specific primer. The arrow and arrowhead on the right indicate the primer-extended products.

A

P0-GFP

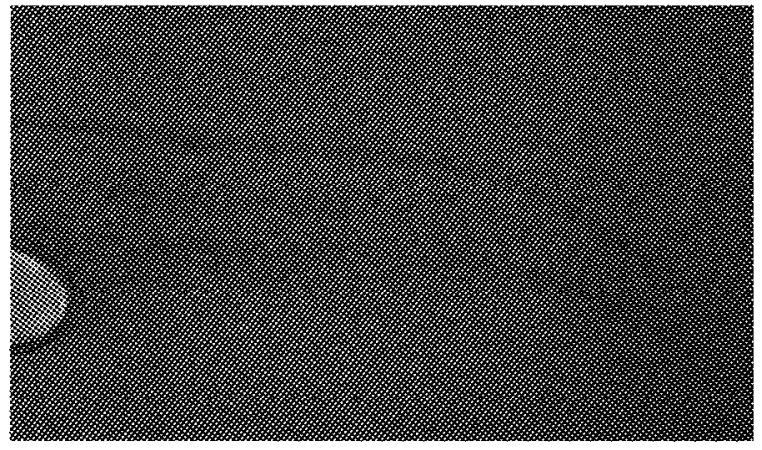

B

\section{Pvegf-GFP}

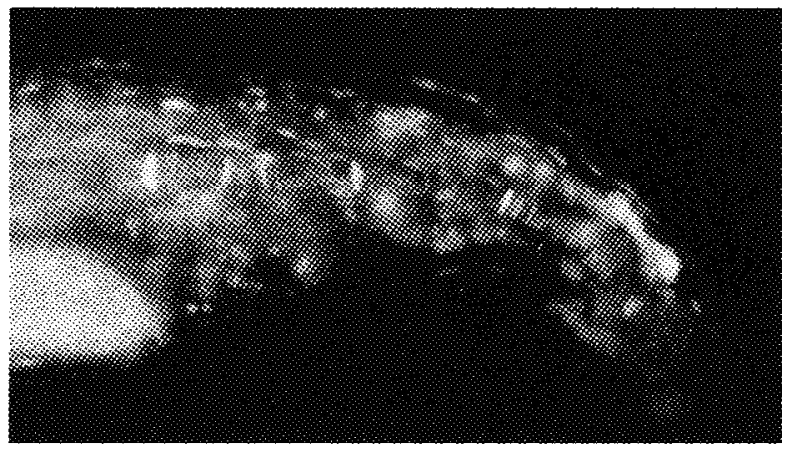

Figure 5. Strong promoter activity of DNA fragment containing upstream and flanking regions of the transcription initiation site. (A) Control zebrafish embryo microinjected with $\mathrm{P}_{0}$-GFP DNA. P here indicates promoter, and 0 indicates zero promoter sequences. (B) GFP expression from zebrafish embryo microinjected with $\mathrm{P}_{\text {vegf }}$-GFP DNA. The embryos are about $24 \mathrm{~h}$ post-fertilization. $\mathrm{P}_{\text {vegf }}$ here includes flanking DNA sequences from $815 \mathrm{bp}$ upstream to $72 \mathrm{bp}$ downstream of the transcription initiation site. 
To prove the biological functionality of the DNA upstream of this transcription initiation site, an 887-bp genomic DNA fragment flanking this transcription start site was placed in front of the green fluorescent protein (GFP) reporter gene. When this DNA was microinjected into fertilized zebrafish eggs, it directed efficient GFP expression in the zebrafish embryos (Figure 5 ). This proves that the transcription initiation site determined above is correct.

It is important to mention that reverse transcriptase in primer extension assays may also be able to perform $\mathrm{C}$ tailing at the $5^{\prime}$ end of the extended first cDNA. In this regard, when mapping the transcription initiation site by primer extension assays, the extended products could be a few nucleotides longer than the real $5^{\prime}$-end nucleotide of the mRNA. So it may not be precisely accurate when the transcription initiation site is determined using this method without directly sequencing the primer-extended products and comparing it to the genomic sequences. Considering this factor, the SMART cDNA synthesis PCR method used here should be as accurate as the primer extension assay in determining the $5^{\prime}$ end of the mRNA. As in most gene regulation studies, knowing the approximate location of the transcription initiation site is sufficient to isolate the promoterenhancer sequences of a gene. In this regard, both SMART cDNA PCR and primer extension assay should be accurate enough for these studies.

It is noted that when we used a modified oligo(dT) primer from Clontech Laboratories (5'-AAGCAGTGGTAACAACGCAGAGATCT $_{(30)} \mathrm{N}_{(-1)} \mathrm{N}-$ $\left.3^{\prime}\right)$ to synthesize the first-strand cDNA and then amplified the cDNA product using gene-specific (GS1 or GS2) primer, we could not obtain any vegfspecific PCR products directly. Instead, a DNA smear of various sizes was obtained, making it impossible to analyze the PCR products directly (data not shown). Southern analysis of the PCRamplified products also showed no vegf-positive DNAs (data not shown). So, using the gene-specific primer to initiate the first-strand cDNA synthesis seems to be pivotal in our hands for the SMART cDNA synthesis PCR method to be successful.
Recently, the SMART cDNA synthesis PCR was modified to reduce the background nonspecific PCR products (3). In this work, we used the earlier SMART cDNA synthesis PCR method. This may explain the multiple bands in Figure 2, lane 3. Whether it is possible to obtain gene-specific PCR product of a low expression gene using the newly optimized SMART PCR method and modified oligo(dT) to initiate the firststrand cDNA synthesis is not known.

In conclusion, the SMART cDNA synthesis PCR method can be used as an alternative method to accurately determine the transcription start site of a gene. Compared to primer extension, the SMART cDNA synthesis PCR method is an easier assay to perform. This method also provides the advantage of not having to find primers very close to the $5^{\prime}$ end of the mRNA. A primer quite far away from the $5^{\prime}$ end can also be used to map the $5^{\prime}$ end of the mRNA accurately ( 950 bases in this case). This can be quite useful when working with novel genes and the location of the transcription initiation site has not been estimated using other methods. Additionally, the amplification nature of PCR can increase the gene-specific cDNA many orders of magnitude. This makes the procedure less difficult even when the gene of interest is expressed at a very low level in the cells. Furthermore, it is a general practice that a series of RT-PCR or other assays be performed to confirm the primer extension assay determined transcription initiation site to be the correct one. Using the SMART PCR method, these steps can be omitted.

\section{REFERENCES}

1.Chenchik, A., L. Diachenko, F. Moqadam, V. Tarabykin, S. Lukyanov and P.D. Siebert. 1996. Full-length cDNA cloning and determination of mRNA $5^{\prime}$ and $3^{\prime}$ ends by amplification of adaptor-ligated cDNA. BioTechniques 21:526-534.

2.Chenchik, A, Y.Y. Zhu, L. Diatchenko, R. Li, J. Hill and P.D. Siebert. 1998. Generation and use of high-quality cDNA from small amounts of total RNA by SMART PCR, p. 305-319. In P. Siebert and J. Larrick (Eds.), Gene Cloning and Analysis by RT-PCR. Eaton Publishing, Natick, MA.

3.Matz, M, D. Shagin, E. Bogdanova, S. Lukyanov, L. Diatchenko and A. Chenchik. 1999. Amplification of cDNA ends based on template-switching effect and step-out PCR. Nucleic Acids Res. 20:5173-5179.

4.Schwabe, W, J.E. Lee, M. Nathan, R.H. Xu, K. Sitaraman, M. Smith, R.J. Potter, K. Rosenthal et al. 1998. THERMOSCRIPT TM RT, a new avian reverse transcriptase for hightemperature cDNA synthesis to improve RTPCR. Focus 20:30-33.

We would like to thank Ms. Lihui Liu for providing the 24-h embryonic total RNA. This work was supported by a research Grant No. RP960369 from the National University of Singapore. Address correspondence to Dr. Ruowen Ge, Department of Biological Sciences, Faculty of Science, National University of Singapore, 10 Kent Ridge Crescent, Singapore 119260. Internet:dbsgerw@nus.edu.sg

Received 18 August 1999; accepted 31 January 2000.

Baiwei Gong and Ruowen Ge National University

of Singapore

Singapore 